\title{
Comparación del proceso de innovación en México entre los regímenes proteccionista y de apertura: un análisis econométrico de inestabilidad de parámetros y cambio estructural, 1940-2015
}

\section{Comparison of the Innovation Process in Mexico between the Protectionist and Free Trade Regimes: An Econometric Analysis of Parameter Instability and Structural Change, 1940-2015}

Juan Ignacio Campa Navarro **, Francisco Venegas-Martínez $z^{* * *}$ y Salvador Cruz-Akét****

\begin{abstract}
RESUMEN
En esta investigación se analiza la estabilidad de la dinámica de patentamiento en México durante los periodos de industrialización por sustitución de importaciones (ISI), (1940-1970), y el intercambio liberalizado (IL) (1995-actual) a través de los registros de patentes disponibles. Para este estudio se compara el registro de patentes en ambos periodos, adicionalmente se hace un análisis econométrico de inestabilidad de parámetros y cambio estructural. Nuestro estudio sugiere una continuidad en la propensión a patentar, aunque cambia la composición de este proceso hacia el patentamiento extranjero y empresarial.
\end{abstract}

Palabras clave: Innovación, patentes, propiedad intelectual, México.

Clasificación JEL: O31, O34, Q51.

\begin{abstract}
This research analyzes the stability of the patenting dynamics in Mexico during the periods of industrialization by import substitution (ISI), (1940-1970), and the liberalized exchange (IL) (1995-current) through the records of available patents. For this study, the patent register is compared in both periods, in addition an econometric analysis of parameter instability and structural change is made. Our study suggests a continuity in the propensity to patent, although the composition of this process changes towards foreign and corporate patenting.
\end{abstract}

Keywords: Innovation, patents, intellectual property, Mexico.

JEL Classification: O31, O34, Q51.

\footnotetext{
* Fecha de recepción: 02/07/2017. Fecha de aceptación: 03/12/2018.

** Universidad Autónoma de Barcelona, España. E-mail: xuan.ijnazio@gmail.com. ORCID: 0000-0003-2998-2960.

*** Instituto Politécnico Nacional, México. E-mail: fvenegas1111@yahoo.com.mx. ORCID: 0000-0003-1157-0298.

**** Instituto Politécnico Nacional, México. E-mail: salvador.ake22@gmail.com. ORCID: 0000-0003-1452-377X.
} 


\section{INTRODUCCIÓN}

El régimen de industrialización por sustitución de importaciones (ISI en adelante) y el régimen de liberalización económica o intercambio liberalizado (IL en adelante) han sido dos modelos relevantes en la historia económica contemporánea de México. El régimen de ISI establecido entre 1940 y 1970 fue un modelo de impulso a la industria nacional donde el país tuvo un desempeño económico elevado registrando tasas de crecimiento del 6 por ciento en promedio anual. Este crecimiento se basó en una política de acumulación de capital extensivo donde la modernización industrial pasaba por proteger a las industrias locales de la competencia exterior pero a su vez permitiendo una transferencia importante de tecnologías incorporadas y no incorporadas en inversión extranjera, bienes de capital, máquinas, herramientas, contratos de asistencia técnica, know-how, etcétera.

El modelo ISI mostró limitaciones que, conjuntamente con crisis recurrentes y la globalización, lo llevó a su conclusión. A partir de ello, el gobierno mexicano impulsó una serie de reformas dirigidas a la apertura de los mercados, privatización de bienes públicos y desregulación.

Con la membresía de México al Tratado de Libre Comercio de América del Norte (TLCAN) en 1994 se desarrolló formalmente un modelo económico nuevo con políticas e incentivos diametrales al modelo de ISI y que continúa en el momento actual.

El cambio de modelo condujo a un proceso de reconversión industrial. En este periodo la economía crece a tasas promedio anual del 2.5 por ciento. La modernización del aparato productivo ancló sus requerimientos de producción al exterior y a la integración en cadenas productivas globales con fuerte contenido de importación tecnológica. ${ }^{1}$

1 Para una revisión de la historia económica de México a lo largo del siglo XX consultar los trabajos de Cárdenas, 2015; Márquez, 2014; Kuntz, 2010; Moreno y Ros, 2009; Schettino, 2007. 
En este trabajo se postula que el ritmo de patentamiento en México se ha mantenido a lo largo de ambos regímenes. Del mismo modo, se postula que la composición de los agentes económicos que promueven estas patentes se ha mantenido en ambos periodos. Para comprobar ambas afirmaciones se analizan los registros de patentes concedidas en México, se elabora un perfil aproximado del tipo de agentes económicos relacionados con el patentamiento a lo largo de ambos modelos económicos, ${ }^{2}$ (ISI e IL).

A lo largo del trabajo se muestra que existe un predominio de patentes en manos de no residentes, (corporaciones transnacionales) durante ambos regímenes económicos, También se hace notar que el patentamiento se ha conservado en los mismos campos tecnológicos. Esto sugiere que la dirección del desenvolvimiento tecnológico mexicano no se ha alterado significativamente durante los regímenes.

Aunque el patentamiento es un indicador de cambio tecnológico, éste ha sido estudiado principalmente en países desarrollados, con escasas investigaciones en países en desarrollo (Sáiz, 2002). En el caso de México, el estudio del tema se ha centrado en la política tecnológica nacional; al sistema nacional de innovación; la dependencia tecnológica o al estudio de casos específicos, entre otros, pero sin ahondar en las patentes como objeto de estudio (Gauss, 2011; Dutrénit, et al., 2010; Corona, 2004).

Gran parte de los estudios antes mencionados carecen de un análisis formal de los patrones del desarrollo tecnológico nacional, excepción hecha a los trabajos de Guzmán, López y Venegas-Martínez (2012). El contraste econométrico de los patrones de patentamiento entre los dos principales regímenes de crecimiento económico mexicano hace novedosa esta investigación.

La literatura sugiere que el registro de patentes en México ha buscado atraer tecnologías del exterior, por lo que el patentamiento nacional ha reflejado dependencia tecnológica como resultado de la política de patentes y las expectativas de beneficios que ha ofrecido la política económica coyuntural (Aboites y Soria, 2008; Beatty, 2015, 2001; Mendoza, 2014).

\footnotetext{
Sin intentar que todos los estudiosos estén de acuerdo, en términos generales, la innovación es una idea, concepto, bien o servicio nuevo o mejorado. La tecnología es un conjunto de conocimientos, técnicas, herramientas, instrumentos, máquinas, procedimientos, organizaciones, métodos y sistemas para producir o transformar bienes y servicios. La innovación tecnológica combina los dos aspectos anteriores. Para cerrar esta cuestión, la ciencia es un conjunto de conocimientos sistemáticamente organizados.
} 
La relación entre el cambio técnico y la economía ha sido ampliamente debatida en Economía. Algunos estudios muestran cómo las tecnologías novedosas llegan a ser factores determinantes del desarrollo económico, o enfoque de oferta tecnológica (Kuznets, 1930; Romer, 1990; Grossman y Helpman, 1991).

Otros trabajos muestran que los procesos de invención e innovación siguen a las condiciones económicas (crecimiento de la producción, inversiones, etcétera), o enfoque de demanda tecnológica (Schmookler 1966; Sokoloff, 1988; Ortiz Villajos, 1999). ${ }^{3}$ Esta investigación se pliega a esta segunda rama teórica.

Este trabajo se encuentra organizado en los siguientes apartados. En la sección I se presenta el método de análisis y las fuentes de los datos. En la sección II se examina la evolución del agregado de las patentes concedidas en territorio nacional, haciendo una primera aproximación al análisis del dinamismo de la actividad de patentamiento bajo dos políticas económicas de distinta naturaleza.

En la sección III se identifica el origen territorial de las patentes. En la sección IV se examina el tipo de persona legal, física o moral, propietaria de las patentes. En la sección $\mathrm{V}$ se muestran los campos tecnológicos donde se agrupa el patentamiento nacional con el fin de delinear la dirección del desarrollo tecnológico. En la sección VI se realiza un análisis econométrico de estabilidad del patrón de las patentes, revelando su intensidad y orientación. En la última sección se presentan las conclusiones.

\footnotetext{
Las patentes como un indicador de actividad tecnológica y su relación con las variables económicas también han sido objeto de análisis y debate examinado en trabajos relativamente recientes de historiadores que sugieren que las patentes son indicadores de pautas de inversión en tecnologías donde las expectativas de beneficio en países e industrias conforman los incentivos de la propensión a patentar (Beatty, 2001; Ortiz-Villajos, 1999; Saiz, 1999). Además de otros enfoques que suponen que las tecnologías y patentes dependen de la especificidad, cooperación y coordinación que proveen los medios institucionales para su protección e implementación (Mokyr, 1977; Freeman, 1987). Los trabajos que ven a la tecnología como un problema de información (Arrow, 1962) o donde los procesos de innovación son resultado de constructos y percepciones subjetivas y de aprendizaje abstracto y de experiencias sensibles como el know-how (North, 2005; Pavit, 1985).
} 


\section{MÉTODO Y FUENTES}

El uso y análisis de los datos de patentes como indicadores tecnológicos o económicos presenta ventajas y desventajas como cualquier indicador respecto de otros. Las patentes conforman acervos de información tecnológica de cobertura espacial y temporal amplia que facilita explorar el estudio presente o pasado de las actividades tecnológicas. Proporcionan datos bibliográficos que permiten conocer quién desarrolla los esfuerzos en investigación y desarrollo, conocer el estado de la técnica o esbozar las posibles direcciones del cambio técnico.

Por otro lado, las patentes tienen las desventajas de no reflejar toda la actividad inventiva de una economía pues no todas las invenciones se patentan o son patentables. Existen aspectos tecnológicos como los cambios organizaciones o el aprender haciendo que no llegan a patentarse aun cuando tengan gran impacto económico en los procesos productivos. Además, no todo lo que se inventa y patenta se explota o comercializa, y hay inventos que se explotan sin la protección de la propiedad industrial (Archibugi, 1996; Griliches, 1992; Basberg, 1987; Pavitt, 1984).

En general, se acepta que la información contenida en las patentes puede ser útil para la elaboración de indicadores parciales de esfuerzos de invención o innovación así como de pautas de inversión en tecnologías novedosas, lo cual por tanto asumimos en este trabajo para perfilar la innovación tecnológica nacional y su análisis en los periodos indicados.

Los datos de patentes utilizados en este estudio en gran parte se construyeron a partir de información que proporciona el Instituto Mexicano de la Propiedad Industrial (IMPI). La información estadística comprendida para el periodo de 1940 a 1970 proviene de una base de datos creada por Juan Ignacio Campa para su tesis doctoral de 2016 con información obtenida de los resúmenes de patentes concedidas de la revista "Gaceta de la Propiedad Industrial”. Para el periodo de 1995-2015 los datos son extraídos de los informes de actividades de periodicidad anual que elaboran el IMPI y el CONACYT respectivamente. ${ }^{4}$

4 Consejo Nacional en Ciencia y Tecnología (CONACYT). 
Dada la heterogeneidad de las fuentes, no se cuenta con el mismo nivel de detalle de la información para ambos periodos. Para el segundo periodo (19952015) no se cuenta con información detallada de los inventores individuales, organizaciones no corporativas y empresas que obtuvieron las patentes. Estos datos sólo permiten un agrupamiento por sectores tecnológicos a un nivel de catalogación de dos dígitos con respecto de la Clasificación Internacional de $\mathrm{Pa}$ tentes (CIP).

Debido a la limitante anterior, la información se agrupa de tal forma que ambos periodos sean comparables. Este nivel de agregación impide identificar la evolución tecnológica y los avances cualitativos de los productos y procesos contenidos en las patentes.

\section{ACTIVIDAD DEL PATENTAMIENTO COMPARADA}

En este apartado mostramos la evolución de la actividad de patentamiento global en perspectiva comparada entre el periodo de industrialización por sustitución de importaciones y el periodo de mercado abierto. En el apartado del análisis estructural se presenta la trayectoria del patentamiento en el periodo largo entero de 1940-2015. En la gráfica 1 vemos que el nivel de patentes registradas en México entre 1940 y 1970 fue relativamente bajo y no fue hasta la fase tardía del régimen cuando alcanzó su umbral máximo. Por otra parte, al comienzo del periodo de IL el patentamiento era menor que el registrado en la fase última de la ISI, aunque el patentamiento registra una tendencia ascendente más temprana.

Se observa también el ritmo de crecimiento diferenciado del patentamiento entre la ISI y el IL (7.0 por ciento y 5.0 por ciento respectivamente) aun cuando el crecimiento económico es sustancialmente distinto en ambos periodos (6.2 por ciento y 2.9 por ciento respectivamente). Esto sugiere una falta de relación entre el patentamiento y el crecimiento económico. 
Gráfica 1. Patentes concedidas. ISI (1940-1970) e IL (1995-2015).

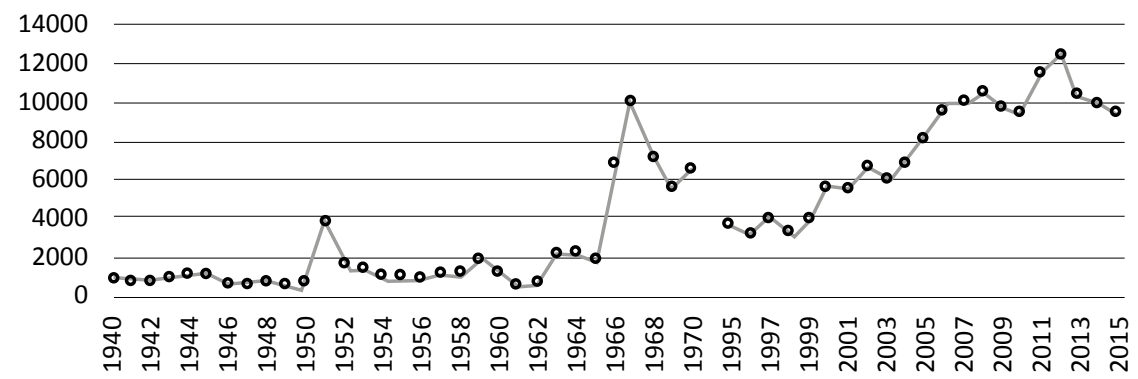

Fuente: OMPI (1983) e IMPI (2016).

El cambio de incentivos asociado al cambio de modelo de crecimiento no parece haber alterado significativamente la dinámica del patentamiento. Para identificar esta situación, en la sección VI, se realiza un análisis econométrico de la estabilidad del patrón tecnológico bajo los dos regímenes económicos.

Resulta llamativa la falta de cambio estructural aun con las reformas al régimen de patentes mexicano y el establecimiento del Tratado de Cooperación en materia de patentes (РСТ) en 1995. Este tratado ha estimulado la propensión a registrar adelantos tecnológicos de origen extranjero dentro del territorio nacional, en particular después de 2005, lo cual coincide con la introducción de una serie de reformas al sistema de salud en contra de la protección de los medicamentos genéricos (Shadlen, 2012).

\section{ORIGEN DE LA PROPIEDAD DE LAS PATENTES}

La distribución de las patentes en propiedad de mexicanos y extranjeros era relativamente equitativa al inicio de la ISI. Esta situación se revirtió a lo largo de la etapa proteccionista, cuadro 1 . Al término del régimen en 1970 el volumen de patentes de mexicanos fue sólo del 5 por ciento mientras que los extranjeros se apropiaron del 95 por ciento de todas las patentes concedidas. La tendencia de participaciones en el registro de patentes persistió durante todo el periodo de apertura. De tal magnitud que a fin del periodo los mexicanos han conservado entre 2 y 4 por ciento del patentamiento total durante dicha etapa. 
De esta caracterización primera resalta una acentuada dependencia tecnológica del exterior, presente durante los dos regímenes económicos. Lo que sugiere que los extranjeros han sabido aprovechar las condiciones e incentivos prevalecientes en ambos periodos para proteger sus tecnologías dentro del territorio nacional. Por contrario, los mexicanos no han propendido por la innovación local pese las oportunidades con los cambios de política económica.

Cuadro 1. Patentes registradas por mexicanos y extranjeros, 1940-1970 y 1995-2015.

\begin{tabular}{cccccc}
\hline \multirow{2}{*}{ Año } & $\begin{array}{c}\text { Total } \\
\text { patentes }\end{array}$ & Residentes & $\%$ & No residentes & $\%$ \\
\hline \multicolumn{7}{c}{ Régimen ISI } \\
\hline 1940 & 863 & 438 & 51 & 425 & 49 \\
\hline 1950 & 646 & 214 & 33 & 419 & 65 \\
\hline 1960 & 1066 & 96 & 9 & 970 & 91 \\
\hline 1970 & 6527 & 342 & 5 & 6185 & 95 \\
\hline 1995 & 3538 & 148 & 4 & 3390 & 96 \\
\hline 2000 & 5519 & 118 & 2 & 5401 & 98 \\
\hline 2005 & 8098 & 131 & 2 & 7967 & 98 \\
\hline 2010 & 9399 & 229 & 2 & 9170 & 96 \\
\hline 2015 & 9338 & 410 & 4 & 8928 & \\
\hline
\end{tabular}

Fuente: OMPI (1983), IMPI (2016) y Campa (2016).

\section{IDENTIDAD DE LOS PROPIETARIOS}

Presentamos una caracterización de los mexicanos y extranjeros propietarios de las patentes por el tipo de identidad o personalidad legal que los distinguía. Durante la ISI los mexicanos individuos inventores eran los que poseían más derechos de propiedad, cuadro 2 . Al inicio del periodo abarcaban el 94 por ciento de las patentes y al término de la etapa poseían un 82 por ciento de la participación.

Las empresas mexicanas tuvieron una participación discreta con un 17 por ciento al fin del periodo. En esta etapa la figura de organizaciones no corporativas tuvo una participación residual. Durante el proteccionismo las empresas extranjeras concentraron el registro de patentes a lo largo del periodo. Al inicio poseían el 58 por ciento y al final el 91 por ciento del patentamiento total. A diferencia, los individuos extranjeros fueron declinando su participación a un 8 por ciento del total de patentes en 1970 . Las organizaciones no corporativas extranjeras tuvieron un registro de patentes también marginal. 
Cuadro 2. Identidad legal de los propietarios de patentes mexicanos y extranjeros.

\begin{tabular}{|c|c|c|c|c|c|c|c|}
\hline Año & $\begin{array}{c}\text { Total patentes } \\
\text { concedidas }\end{array}$ & Individuos & $\%$ & Corporaciones & $\%$ & $\begin{array}{l}\text { Organizaciones } \\
\text { no corporativas }\end{array}$ & $\%$ \\
\hline \multicolumn{8}{|c|}{ Régimen ISI } \\
\hline \multicolumn{8}{|c|}{ Mexicanos } \\
\hline 1940 & 438 & 413 & 94 & 25 & 6 & 0 & 0 \\
\hline 1970 & 342 & 280 & 82 & 59 & 17 & 3 & 1 \\
\hline \multicolumn{8}{|c|}{ Extranjeros } \\
\hline 1940 & 425 & 176 & 42 & 248 & 58 & 1 & 0 \\
\hline 1970 & 6185 & 493 & 8 & 5653 & 91 & 39 & 1 \\
\hline \multicolumn{8}{|c|}{ Régimen IL } \\
\hline \multicolumn{8}{|c|}{ Mexicanos } \\
\hline 1996 & 116 & 59 & 51 & 37 & 32 & 20 & 17 \\
\hline 2015 & 410 & 123 & 30 & 131 & 32 & 156 & 38 \\
\hline \multicolumn{8}{|c|}{ Extranjeros } \\
\hline 1996 & 3070 & 141 & 5 & 2,923 & 95 & 6 & 0 \\
\hline 2105 & 8934 & 243 & 3 & 8,593 & 96 & 98 & 1 \\
\hline
\end{tabular}

Fuente: Campa (2018).

La tendencia del patentamiento de mexicanos y de extranjeros mostró variaciones durante la etapa de apertura. En el caso de los mexicanos, un cambio notorio provino de las organizaciones no corporativas que han emergido significativamente como propietarias de patentes. Al comenzar el periodo en 1995 registraron un 17 por ciento del patentamiento y en 2015 un 38 por ciento, con lo cual ha superado el patentamiento de individuos y de empresas. Gran parte de las patentes registradas corresponden a universidades y centros de investigación públicos nacionales. En el caso de los extranjeros la propensión a patentar fue más estable, manteniéndose relativamente la concentración del patentamiento del sector empresarial extranjero entre un 90 a 96 por ciento del total de patentes a lo largo del periodo IL.

La preeminencia de las empresas extranjeras en el patentamiento durante ambos regímenes económicos es explicable debido a que los incentivos establecidos en ambas etapas favoreció la propensión a patentar por estos agentes. Durante la ISI, la expansión industrial requirió un volumen creciente de capitales que fueron introducidos al país vía inversión extranjera directa e importación de bienes de capital tangible e intangible, los cuales incorporaban la protección de patentes. Con las políticas de apertura, desreglamentación y privatización del régimen IL se ha incentivado el interés del empresariado extranjero en invertir en patentes capturando las ventajas del comercio liberalizado y sus rentas asociadas. 
Los mexicanos han tenido una participación conservadora en la actividad de patentamiento. Tanto los individuos como las empresas no han respondido favorablemente a los incentivos de cada etapa económica. Sin embargo, las organizaciones no corporativas han emergido como un actor importante en la actividad de patentamiento nacional en la etapa IL. Una explicación de la emergencia de este colectivo es el esfuerzo de universidades, centros e institutos de investigación y desarrollo locales en desarrollar políticas propias sobre registro de patentes y transferencia de tecnología, desde los años ochenta.

Tales políticas sobre patentes y de contratos de transferencia tecnológica se han encuadrado a su vez dentro de los avances que se han concretado en materia de formulación e implementación del sistema nacional de innovación mexicano que ha incentivado, a partir de la década de los noventa, una política más sólida de vinculación entre la academia y los centros de investigación con el sector empresarial privado en esfuerzos de $\mathrm{I}+\mathrm{D}+\mathrm{I}$ dando paso a diferentes modalidades de contratación tecnológica incluida la transferencia de patentes (Villavicencio, 2012; Casalet, 2012; Dutrénit, et al., 2010; García y Heijs, 2012; y Casas y Luna, 1997).

\section{PATENTES Y SECTORES TECNOLÓGICOS}

En México los procesos de innovación no han sido muy diferentes entre ambos regímenes económicos; para más detalles véanse cuadros 3 y 4 . El perfil tecnológico conformado por los mexicanos durante la ISI muestra que las patentes se concentraron en los campos relacionados con "necesidades corrientes de la vida"; "técnicas industriales diversas, transportes" y "mecánica, iluminación". Aunque las participaciones de estos campos mostraron fluctuaciones ligeras de posición mantuvieron la tendencia de concentración del patentamiento. En la etapa de IL básicamente se mantuvo una estructura similar salvo que el campo de "química, metalurgia" se posicionó en un grado más alto desplazando al campo de "mecánica", ver cuadro 3.

En 1940 el campo de "necesidades corrientes de la vida" abarcó el 32 por ciento del total de patentes y en 2015 se comprendió un 29 por ciento. El campo de "técnicas industriales diversas, transportes" mantuvo una presencia de 18 por ciento y 20 por ciento en los años antes indicados y el campo de "mecánica" concentró el 15 por ciento pero luego mostró un descenso constante a lo largo de los dos periodos hasta situarse en un 10 por ciento, mismos años. Por contrario, el campo de "química; metalurgia" mantuvo una participación debajo del campo 
de "mecánica" durante la ISI, pero en el régimen de IL prácticamente duplicó su participación pasando de un 10 por ciento en 1940 a 19 por ciento en 2015 a fin del periodo.

Cuadro 3. Distribución por campos tecnológicos de patentes de mexicanos.

\begin{tabular}{|c|c|c|c|c|c|c|c|c|}
\hline Campo tecnológico & 1940 & $\%$ & 1950 & $\%$ & 1960 & $\%$ & 1970 & $\%$ \\
\hline a) necesidades corrientes de la vida & 138 & 31.5 & 53 & 24.9 & 23 & 24.2 & 86 & 25.4 \\
\hline b) técnicas industriales diversas; transportes & 80 & 18.3 & 50 & 23.5 & 26 & 27.4 & 70 & 20.1 \\
\hline c) química; metalurgia & 44 & 10.0 & 23 & 10.3 & 11 & 11.6 & 30 & 8.6 \\
\hline d) textiles; papel & 25 & 5.7 & 13 & 6.1 & 8 & 8.4 & 19 & 5.6 \\
\hline e) construcciones fijas & 31 & 7.1 & 17 & 8.0 & 5 & 5.3 & 40 & 11.8 \\
\hline $\begin{array}{l}\text { f) mecánica; iluminación; calefacción; } \\
\text { armamento; voladura }\end{array}$ & 66 & 15.1 & 24 & 11.3 & 10 & 9.5 & 46 & 13.3 \\
\hline g) física & 35 & 8.0 & 22 & 10.3 & 3 & 3.2 & 28 & 8.3 \\
\hline h) electricidad & 19 & 4.3 & 12 & 5.6 & 10 & 10.5 & 23 & 6.8 \\
\hline Total & 438 & 100 & 214 & 100 & 96 & 100 & 342 & 100 \\
\hline Campo tecnológico & 1995 & $\%$ & 2000 & $\%$ & 2005 & $\%$ & 2015 & $\%$ \\
\hline a) necesidades corrientes de la vida & 37 & 25.0 & 23 & 19.5 & 41 & 31.3 & 118 & 28.8 \\
\hline b) técnicas industriales diversas; transportes & 23 & 15.5 & 31 & 26.3 & 22 & 16.8 & 82 & 20.0 \\
\hline c) química; metalurgia & 31 & 20.9 & 31 & 26.3 & 27 & 20.6 & 79 & 19.3 \\
\hline d) textiles; papel & 2 & 1.4 & 1 & 0.8 & 0 & 0.0 & 2 & 0.5 \\
\hline e) construcciones fijas & 27 & 18.2 & 15 & 12.7 & 15 & 11.5 & 24 & 5.9 \\
\hline $\begin{array}{l}\text { f) mecánica; iluminación; calefacción; } \\
\text { armamento; voladura }\end{array}$ & 17 & 11.5 & 7 & 5.9 & 9 & 6.9 & 42 & 10.2 \\
\hline g) física & 7 & 4.7 & 4 & 3.4 & 11 & 8.4 & 49 & 12.0 \\
\hline h) electricidad & 4 & 2.7 & 6 & 5.1 & 6 & 4.6 & 14 & 3.4 \\
\hline Total & 148 & 100 & 118 & 100 & 131 & 100 & 410 & 100 \\
\hline
\end{tabular}

Fuente: Campa (2018). 
Cuadro 4. Distribución por campos tecnológicos de patentes extranjeros.

\begin{tabular}{|c|c|c|c|c|c|c|c|c|}
\hline Campo tecnológico & 1940 & $\%$ & 1950 & $\%$ & 1960 & $\%$ & 1970 & $\%$ \\
\hline a) necesidades corrientes de la vida & 84 & 19.8 & 70 & 16.7 & 124 & 12.8 & 811 & 13.1 \\
\hline b) técnicas industriales diversas; transportes & 70 & 16.5 & 67 & 16.0 & 159 & 16.4 & 1018 & 16.5 \\
\hline c) química; metalurgia & 79 & 18.6 & 99 & 23.6 & 385 & 39.7 & 2315 & 37.4 \\
\hline d) textiles; papel & 30 & 7.1 & 27 & 6.4 & 69 & 7.1 & 335 & 5.4 \\
\hline e) construcciones fijas & 34 & 8.0 & 14 & 3.3 & 52 & 5.4 & 224 & 3.6 \\
\hline $\begin{array}{l}\text { f) mecánica; iluminación; calefacción; } \\
\text { armamento; voladura }\end{array}$ & 61 & 14.4 & 36 & 8.6 & 83 & 8.6 & 704 & 11.4 \\
\hline g) física & 23 & 5.4 & 31 & 7.4 & 34 & 3.5 & 302 & 4.9 \\
\hline h) electricidad & 44 & 10.4 & 75 & 17.9 & 64 & 6.6 & 476 & 7.7 \\
\hline Total & 425 & 100 & 419 & 100 & 970 & 100 & 6185 & 100 \\
\hline Campo tecnológico & 1995 & $\%$ & 2000 & $\%$ & 2005 & $\%$ & 2015 & $\%$ \\
\hline a) necesidades corrientes de la vida & 490 & 14.5 & 1579 & 29.2 & 1946 & 24.4 & 3051 & 34.2 \\
\hline b) técnicas industriales diversas; transportes & 693 & 20.4 & 1306 & 24.2 & 1359 & 17.1 & 1761 & 19.7 \\
\hline c) química; metalurgia & 1119 & 33.0 & 1348 & 25.0 & 2055 & 25.8 & 1319 & 14.8 \\
\hline d) textiles; papel & 73 & 2.2 & 87 & 1.6 & 192 & 2.4 & 80 & 0.9 \\
\hline e) construcciones fijas & 76 & 2.2 & 89 & 1.6 & 128 & 1.6 & 413 & 4.6 \\
\hline $\begin{array}{l}\text { f) mecánica; iluminación; calefacción; } \\
\text { armamento; voladura }\end{array}$ & 293 & 8.6 & 289 & 5.4 & 579 & 7.3 & 532 & 6.0 \\
\hline g) física & 269 & 7.9 & 262 & 4.9 & 725 & 9.1 & 832 & 9.3 \\
\hline h) electricidad & 377 & 11.1 & 441 & 8.2 & 983 & 12.3 & 940 & 10.5 \\
\hline Total & 3390 & 100 & 5401 & 100 & 7967 & 100 & 8928 & 100 \\
\hline
\end{tabular}

Fuente: Campa (2018). 
Los extranjeros concentraron sus patentes en campos de "técnicas industriales diversas, transportes"; "necesidades corrientes de la vida" y "química, metalurgia". Similar que con el grupo de mexicanos las participaciones del patentamiento de extranjeros mostraron fluctuaciones pero resaltó una tendencia de concentración. Tanto en el régimen de la ISI como en el de IL, los tres campos tecnológicos mencionados abarcaron más patentes. En 1940 la participación acumulada fue del 55 por ciento del total de patentes y en 2015 alcanzó un 77 por ciento, ver cuadro 4.

El campo de "técnicas industriales diversas, transportes" fue el que mostró una tendencia más consistente. Este campo mantuvo una participación relativa del 17 por ciento en 1940 y 22 por ciento en 2015. El campo de "necesidades corrientes de la vida" muestra tendencias opuestas entre periodos. Durante la ISI su participación decayó del 20 por ciento en 1940 al 13 por ciento en 1970. Por contrario, durante la etapa IL el campo se recuperó de un 15 por ciento en 1995 al 38 por ciento en 2015. El campo de "química, metalurgia" también muestra una inflexión en la tendencia. Durante la época proteccionista aumenta del 19 por ciento al 37 por ciento, pero bajo el periodo de intercambio liberalizado este campo declina su participación del 33 por ciento en 1995 a un 17 por ciento en el año 2015. Estas tendencias muestran que el patentamiento de los extranjeros, similar a la propensión a patentar de los mexicanos, han sido relativamente estables revelando también un perfil de la innovación nacional poco dinámico e intensivo.

\section{ANÁLISIS ECONOMÉTRICO DE ESTABILIDAD DEL PATRÓN TECNOLÓGICO}

A lo largo de la investigación, la hipótesis principal es que el ritmo de patentamiento global ha sido prácticamente el mismo a lo largo de ambos periodos (IL-ISI). Para verificarlo, se hará uso de tres metodologías complementarias que aportan evidencia empírica sobre la estabilidad estructural del patentamiento en México. La primera es una prueba de estabilidad estructural de Chow (1960). La segunda consiste en una comparación estadística de los parámetros de la regresión de serie de tiempo del registro de patentes cuando están separados ambos regímenes (ISI-IL) y cuando se toma la muestra completa. Por último, se realizan varias de pruebas de estabilidad estructural de Quandt-Andrews con cortes muestrales al 15 por ciento, 30 por ciento y 45 por ciento de la muestra. 
El análisis econométrico se realiza sobre una serie de tiempo de observaciones anuales del número total de patentes en México entre 1940 y 2014. Esta serie de tiempo (SE_PATENTES_NIV) fue sometida a una prueba de estacionariedad KPSS, Kwiatkowski, et al. (1992), con tendencia e intercepto como lo sugiere la gráfica 1. La prueba se muestra en el cuadro 5 y se observa que la serie es estacionaria al 1 por ciento.

Cuadro 5. Prueba KPSS para la serie de patentes concedidas.

\begin{tabular}{|c|c|c|}
\hline \multicolumn{3}{|c|}{ Hipótesis nula: SE_PATENTES_NIV es estacionaria } \\
\hline \multicolumn{3}{|c|}{ Ancho de banda: 6 (Newey-West) usando el kernel de Bartlett } \\
\hline \multicolumn{2}{|c|}{ Exógenas: constante, tenencia lineal } & Estadístico lm \\
\hline \multicolumn{2}{|c|}{ Estadístico Kwiatkowski-Phillips-Schmidt-Shin } & 0.164417 \\
\hline \multirow[t]{3}{*}{ Valores críticos asintóticos*: } & $1 \%$ & 0.216 \\
\hline & $5 \%$ & 0.146 \\
\hline & $10 \%$ & 0.119 \\
\hline
\end{tabular}

Fuente: cálculos propios con Eviews 9.0 con base en series de datos tomados de OMPI (1983) e IMPI (2016).

Una vez garantizada la estacionariedad de la serie se muestran los modelos ARMA $(2,1)$ aplicados tanto a la muestra completa (cuadro 6) como a la partición correspondiente al régimen ISI (cuadro 7) y al periodo de la IL (cuadro 8), así como sus respectivas gráficas de ajuste. 
Cuadro 6. Modelo ARMA(2,1) 1940-2014.

\begin{tabular}{|lrllr|}
\hline \multicolumn{5}{|c|}{ Variable dependiente: SE_PATENTES_NIV } \\
\hline \multicolumn{5}{|c|}{ Método: ARMA Máxima verosimilitud (OPG-BHHH) } \\
\hline Muestra: 1940 2014 & Observaciones incluídas: 75 \\
\hline Variable & Coeficiente & Error std. & Estad. T & \multicolumn{1}{c|}{ Prob. } \\
\hline @TREND & 184.614 & 20.81713 & 8.868368 & 0 \\
\hline AR(2) & 0.59939 & 0.110427 & 5.427947 & 0 \\
\hline MA(1) & 0.591846 & 0.077218 & 7.664572 & 0 \\
\hline SIGMASQ & 3363268 & 309443.2 & 10.86877 & 0 \\
\hline R-Cuad & 0.843956 & Media Var. Dep. & 6741.77 \\
\hline R-Cuad aj & 0.837363 & S.D. Var. Dep. & 4673.82 \\
\hline S.E. reg. & 1884.873 & Criterio de Akaike & 17.984 \\
\hline RSS & $2.52 E+08$ & Criterio Schwarz & 18.107 \\
\hline Log ver. & -670.4114 & Criterio H-Q & 18.03365 \\
\hline DW & 2.044538 & \multicolumn{2}{c}{} \\
\hline R inv ar & 0.77 & -0.77 & R inv ma & -0.59 \\
\hline
\end{tabular}

Gráfica 2. Ajuste arma(2,1) 1940-2014.

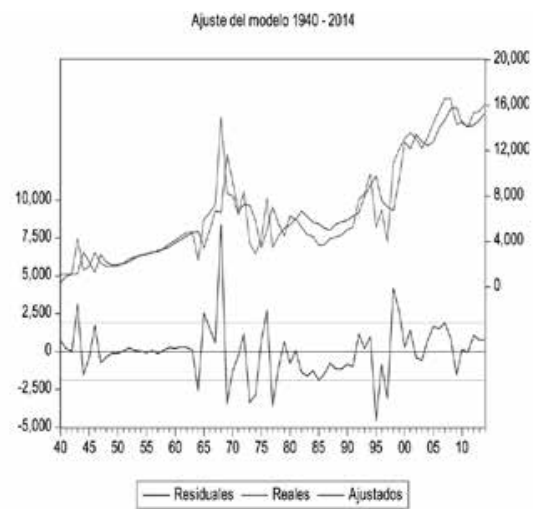

Fuente: cálculos propios con Eviews 9.0 con base en series de datos tomados de OMPI (1983) e IMPI (2016).

Cuadro 7. Modelo ARMA(2,1) 1940-1996.

\begin{tabular}{|lr|rlr|}
\hline \multicolumn{5}{|c|}{ Variable dependiente: SE_PATENTES_NIV } \\
\hline \multicolumn{5}{|c|}{ Método: ARMA Máxima verosimilitud (opg - bhhh) } \\
\hline Muestra: 19401996 & \multicolumn{3}{c|}{ Observaciones incluídas: 75} \\
\hline Variable & Coef & Error std. & Estad. T & Prob. \\
\hline @TREND & 139.6363 & 26.11868 & 5.346226 & 0 \\
\hline AR(2) & 0.4779 & 0.20124 & 2.374782 & 0.0212 \\
\hline MA(1) & 0.500701 & 0.134935 & 3.710683 & 0.0005 \\
\hline SIGMASQ & 3309507 & 340351 & 9.723804 & 0 \\
\hline R-cuad & 0.47254 & Media Var. Dep. & 4572.982 \\
\hline R-cuad aj & 0.442683 & s.d. Var. Dep. & 2527.145 \\
\hline S.E. reg. & 1886.606 & Criterio de Akaike & 18.00022 \\
\hline RSS & $1.89 E+08$ & Criterio Schwarz & 18.14359 \\
\hline Log ver. & -509.0063 & Criterio h-q & 18.05594 \\
\hline DW & 2.078518 & \\
\hline R inv ar & 0.69 & -0.69 R inv ma \\
\hline
\end{tabular}

Gráfica 3. Ajuste ARMA(2,1) 1940-1996.

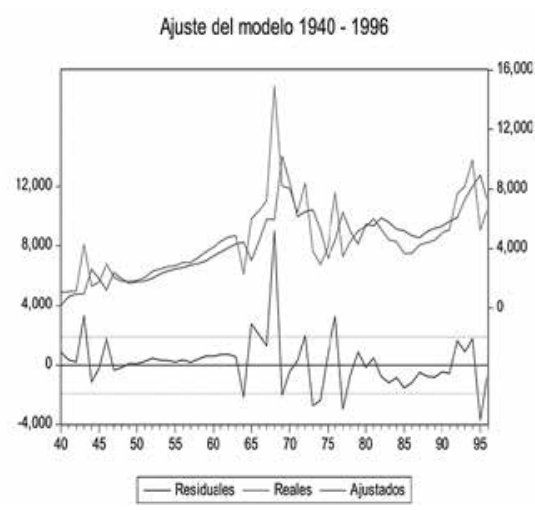

Fuente: cálculos propios con Eviews 9.0 con base en series de datos tomados de OMPI (1983) e IMPI (2016). 
Cuadro 8. Modelo ARMA(2,1) 1940-1996.

\begin{tabular}{|lc|ccc|}
\hline \multicolumn{5}{|c|}{ Variable dependiente: SE_PATENTES_NIV } \\
\hline \multicolumn{5}{|c|}{ Método: ARMA Máxima verosimilitud (opg - bhhh) } \\
\hline Muestra: 19972014 & \multicolumn{3}{c|}{ Observaciones incluídas: 75} \\
\hline Variable & Coef & Error std. & Estad. T & Prob. \\
\hline @TREND & 197.8039 & 43.6335 & 4.533304 & 0.0005 \\
\hline AR(2) & 0.579409 & 0.297799 & 1.945637 & 0.0721 \\
\hline MA(1) & 0.775056 & 0.342136 & 2.265342 & 0.0399 \\
\hline SIGMASQ & 3397837 & 1097737 & 3.09531 & 0.0079 \\
\hline R-Cuad & 0.568507 & Media Var. Dep. & 13609.61 \\
\hline R-cuad aj & 0.476044 & s.d. Var. Dep. & 2887.528 \\
\hline S.E. reg. & 2090.131 & Criterio de Akaike & 18.36989 \\
\hline RSS & 6.12 E+07 & Criterio Schwarz & 18.56775 \\
\hline Log ver. & -161.329 & Criterio h-q & 18.39717 \\
\hline DW & 2.151858 & & -0.78 \\
\hline R inv ar & 0.76 & -0.76 R inv ma \\
\hline \multicolumn{5}{|c}{} \\
\hline
\end{tabular}

Gráfica 4. Ajuste ARMA(2,1) 1940-1996

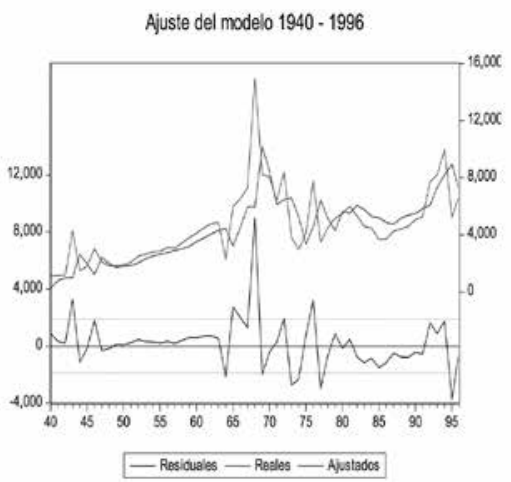

Fuente: cálculos propios con Eviews 9.0 con base en series de datos tomados de OMPI (1983) e IMPI (2016).

El propósito de este ejercicio es comparar los parámetros asociados a cada una de las regresiones. Si éstos resultan estadísticamente similares (se encuentran dentro del mismo intervalo de confianza) se refuerza entonces la evidencia empírica de que no existe una ruptura temporal entre ambos regímenes.

Cuadro 9. Pruebas de media para ambos regímenes comparadas con la regresión global.

\begin{tabular}{llllr}
\hline & \multicolumn{1}{c}{$\mathbf{1 9 4 0 - 1 9 9 6}$} & \multicolumn{1}{c}{$\mathbf{1 9 9 7 - 2 0 1 4}$} \\
\cline { 2 - 5 } & $\mathbf{T}$ & Prob t & T & Prob t \\
\hline @trend & -14.91339 & $7.40182 \mathrm{E}-24$ & 2.617894158 & 0.01075047 \\
\hline $\operatorname{ar}(2)$ & -5.228256 & $1.56737 \mathrm{E}-06$ & -0.58106486 & 0.56298651 \\
\hline ma(1) & -5.849771 & $1.29252 \mathrm{E}-07$ & 4.637469142 & $1.5114 \mathrm{E}-05$ \\
\hline sigmasq & -1.367952 & 0.175523311 & 0.272721355 & 0.7858374 \\
\hline
\end{tabular}

Fuente: cálculos propios con Excel con base en series de datos tomados de OMPI (1983) e IMPI (2016). 
En el cuadro 9 se muestran los resultados de las pruebas de media de dos muestras (dos colas) aplicadas a todos los parámetros asociados en ambos regímenes (ISI en el cuadro 7 e IL en el cuadro 8). Al ser comparadas con los parámetros de la regresión general (parámetros del cuadro 6), se tiene que todos los parámetros, excepto la dispersión (en ambos regímenes) y el término AR(2) en el régimen IL (1997-2014), son estadísticamente iguales (con un nivel de confianza del 5 por ciento), lo que verifica empíricamente la hipótesis de estabilidad en la cantidad anual de patentes registrada en ambos periodos.

Otra prueba asociada a este procedimiento basada en regresiones es la prueba de Chow (prueba F) que compara la diferencia de las sumas de residuales al cuadrado del modelo completo con los modelos particionados con la suma de residuales al cuadrado de los modelos particionados. El uso de esta prueba está condicionado al conocimiento de una fecha específica a partir de la cual se separa la serie de tiempo. En esta investigación se toma 1997 con la finalidad de dar tiempo a la implementación del TLCAN (que entra en vigor en 1994), los resultados de la prueba de Chow son mostrados en el cuadro 10.

Cuadro 10. Prueba de Chow, punto de cambio estructural en 1997 para la serie de registro de patentes en México.

\begin{tabular}{lllr}
\hline \multicolumn{4}{c}{ Prueba de cambio estructural Chow: 1997} \\
\hline \multicolumn{4}{c}{ Hipótesis nula: no hay rompimiento en el punto especificado } \\
\hline \multicolumn{4}{c}{ Muestra de la ecuación: 19402014} \\
\hline Estadístico F & 0.163755 & Prob. $F(4,67)$ & 0.956 \\
\hline Cociente de Log-Verosimilitud & 1.579842 & Prob. Chi-Cuad(4) & 0.8124 \\
\hline Estadístico de Wald & 2.232295 & Prob. Chi-Cuad(4) & 0.6931 \\
\hline
\end{tabular}

Fuente: cálculos propios con Eviews 9.0 con base en series de datos tomados de OMPI (1983) e IMPI (2016).

El cuadro 10 muestra que la prueba de Chow no rechaza la hipótesis nula de la inexistencia del rompimiento estructural en 1997, es decir, que dicha prueba aporta evidencia empírica sobre la estabilidad estructural del patentamiento en México. Es bien conocido que los resultados de la prueba de Chow son discutibles dada la elección del punto de cambio estructural por parte del investigador. Para evitar este problema se muestran en los cuadros 11, 12 y 13 pruebas de Quandt-Andrews, las cuales tienen el mismo objetivo que las pruebas de Chow, pero no suponen un punto de rompimiento específico, sino que toman percentiles determinados y simétricos a lo largo de la muestra para probar la estabilidad estructural. 
El hecho de no tener un punto de rompimiento estructural dificulta el análisis de las distribuciones asintóticas de los estimadores pues, aunque las distribuciones de los estimadores de Wald son conocidas, éstas difieren en función de los puntos de ruptura. De la misma forma, las versiones de la prueba (usando el promedio o la versión del logaritmo natural del promedio del estimador de Wald) dependen del cambio esperado en los parámetros (cambios pequeños o grandes de los parámetros), respectivamente; véanse, por ejemplo, los trabajos de Quandt (1960); Andrews (1993) y Andrews y Ploberger (1994).

Cuadro 11. Prueba de estabilidad de Quandt-Andrews para la serie de otorgamiento de patentes en México al 15 por ciento de la muestra.

\begin{tabular}{lrr}
\hline \multicolumn{3}{c}{ Prueba Quandt-Andrews para rompimiento estructural desconocido } \\
\hline \multicolumn{2}{c}{ Hipótesis nula: sin rompimiento en recorte de 15\% de la muestra } \\
\hline \multicolumn{2}{c}{ Muestra total: 1940 2014 } & Muestra de prueba: 19522003 \\
\hline Rompimientos comparados: 52 & Valor & Prob. \\
\hline Estadístico & 3.680738 & 0.086 \\
\hline Max. Est. Ir f (1998) & 56.49517 & 0 \\
\hline Max. Est. Wald F(1964) & 0.555639 & 0.5329 \\
\hline Estad. Exp Ir f & 24.97543 & 0.5231 \\
\hline Estad. Exp Wald F & 0.8798 & 0 \\
\hline Est. Ave Ir f & 17.43079 & \\
\hline Est. Ave Wald F & & \\
\hline
\end{tabular}

Fuente: cálculos propios con Eviews 9.0 con base en series de datos tomados de OMPI (1983) e IMPI (2016).

Cuadro 12. Prueba de estabilidad de Quandt-Andrews para la serie de otorgamiento de patentes en México al 30 por ciento de la muestra.

\begin{tabular}{lcc}
\hline \multicolumn{3}{c}{ Prueba Quandt-Andrews para rompimiento estructural desconocido } \\
\hline \multicolumn{2}{c}{ Hipótesis nula: sin rompimiento en recorte de 30\% de la muestra } \\
\hline \multicolumn{1}{c}{ Rompimientos comparados: 52} & Muestra de prueba: 19631992 \\
\hline Estadístico & Valor & Prob. \\
\hline Max. Est. Ir f (1998) & 3.289236 & 0.0863 \\
\hline Max. Est. Wald F(1964) & 56.49517 & 0 \\
\hline Estad. Exp Ir f & 0.652502 & 0.349 \\
\hline Estad. Exp Wald F & 25.28757 & 0 \\
\hline Est. Ave Ir f & 1.111877 & 0.3356 \\
\hline Est. Ave Wald F & 11.4965 & 0.008 \\
\hline
\end{tabular}

Fuente: cálculos propios con Eviews 9.0 con base en series de datos tomados de OMPI (1983) e IMPI (2016). 
Cuadro. 13. Prueba de estabilidad de Quandt-Andrews para la serie de otorgamiento de patentes en México al 45 por ciento de la muestra.

\begin{tabular}{lcc}
\hline \multicolumn{3}{c}{ Prueba Quandt-Andrews para rompimiento estructural desconocido } \\
\hline \multicolumn{3}{c}{ Hipótesis nula: sin rompimiento en recorte de 45\% de la muestra } \\
\hline \multicolumn{2}{c}{ Rompimientos comparados: 52} & Muestra de prueba: 1974 1981 \\
\hline Estadístico & Valor & Prob. \\
\hline Max. Est. Ir f (1977) & 2.414657 & 0.1277 \\
\hline Max. Est. Wald F(1977) & 9.053344 & 0.1567 \\
\hline Estad. Exp Ir f & 0.699049 & 0.2528 \\
\hline Estad. Exp Wald F & 3.090732 & 0.2035 \\
\hline Est. Ave Ir f & 1.341399 & 0.242 \\
\hline Est. Ave Wald F & 5.191058 & 0.2591 \\
\hline
\end{tabular}

Fuente: cálculos propios con Eviews 9.0 con base en series de datos tomados de OMPI (1983) e IMPI (2016).

A través de las pruebas de Quandt-Andrews realizadas en esta sección es posible verificar la estabilidad estructural de la serie de tiempo del registro de patentes en México a diferentes particiones de la muestra. En efecto, partiendo de una hipótesis nula de inexistencia de rompimiento estructural, sólo el cuadro 11 muestra un posible rompimiento estructural para 1964, el cual regresa a la tendencia en 1969.

Tal vez el resultado más llamativo de las pruebas Quandt-Andrews es la falta de rompimientos estructurales en los grandes hitos del comercio mexicano: la entrada al GAT y el ingreso al TLCAN. Esta pieza de evidencia empírica muestra que los flujos de comercio no impactaron al desarrollo de las innovaciones, lo que parece contradecir el argumento del derrame tecnológico asociado al comercio, pues el patentamiento sólo presenta una tendencia temporal que no es modificada en ningún punto de la muestra. Por supuesto, este último punto queda como una posible línea de investigación. 


\section{CONCLUSIONES}

La caracterización comparada de la actividad de patentamiento bajo los regímenes de proteccionismo y de mercado abierto sugiere que la innovación tecnológica en México dependió acentuadamente de tecnologías patentadas del extranjero en ambas etapas. Sin embargo, la propensión a patentar de mexicanos y de los extranjeros fue similar en los sectores tecnológicos también durante ambos regímenes, lo que muestra que la innovación tecnológica nacional fue poco dinámica y siguió un patrón parecido en el patentamiento.

La evidencia empírica dada por las pruebas de media de los parámetros de tendencia de la serie completa comparados con los parámetros de tendencia de las series divididas sugiere que no hay cambios de dirección en el modelo de desarrollo tecnológico nacional con la transición de un modelo económico a otro, pero lo que sí ha cambiado es la volatilidad de los procesos, ésta ha aumentado durante la IL. La hipótesis de estabilidad estructural también es fortalecida por los análisis de estabilidad de Chow y de Quandt-Andrews para puntos de ruptura conocidos o no, respectivamente. En ambos casos, las pruebas no rechazan la hipótesis nula de estabilidad estructural. Como aspecto importante de estas pruebas queda el año de 1964, cuando la prueba Q-A detecta un posible cambio estructural que regresa a la tendencia en 1969. 


\section{REFERENCIAS BIBLIOGRÁFICAS}

Aboites, Jaime y Soria, Manuel (2008), Economía del conocimiento y propiedad intelectual. Lecciones para la economía mexicana, Editorial Siglo XXI, México. Andrews, Donald (1993), "Tests for Parameter Instability and Structural Change with Unknown Change Point”, Econometrica, vol. 61, núm. 4, pp. 821-856.

Andrews, Donald y Ploberger, Werner (1994), "Optimal Tests when a Nuisance Parameter is Present Only under the Alternative”, Econometrica, vol. 62, núm. 6, pp. 1383-1414.

Archibugi, Daniele y Pianta, Mario (1996), "Measuring Technological Change Through Patents and Innovation Surveys", Technovation, 16 (9), pp. 451-468.

Arrow, Kenneth (1962), "Economic Welfare and the Allocation of Resources for Invention", en Nelson, Richard (ed.), The Rate and Direction of Inventive Activity, New York, pp. 609-625.

Basberg, Bjørn (1987), "Patents and Measurement of Technological Change: A Survey of the Literature", Research Policy, 16, pp. 131-141.

Beatty, Edward (2015), Technology and Search for Progress in Modern Mexico, University California Press.

(2001), Institutions and investment. The Political Basis of Industrialization in Mexico Before 1911, Stanford University Press, California.

Campa, Juan Ignacio (2016), Patentes en México en la época de la industrialización por sustitución de importaciones, tesis doctoral, Universidad Autónoma de Barcelona.

(2018), "Patentes y desenvolvimiento tecnológico en México: Un estudio comparativo entre la época de industrialización proteccionista y el régimen de apertura", América Latina en la Historia Económica, año 25, número 3, cuarto trimestre.

Cárdenas, Enrique (2015), El largo curso de la economía mexicana. De 1780 a nuestros días, FCE- COLmex, México.

Casalet, Mónica (2012), "Las relaciones de colaboración entre la universidad y los sectores productivos: una oportunidad a construir en la política de innovación", en Carrillo, Jorge; Hualde, Alfredo y Villavicencio, Daniel (coords.), Dilemas de la Innovación en México. Dinámicas sectoriales, territoriales e institucionales, Colegio de la Frontera Norte y Red Temática Complejidad, México, cap. 4.

Casas, Rosalba y Luna, Matilde (1997), Gobierno, academia y empresas en México. Hacia una nueva configuración de relaciones, Plaza y Valdés/IIS-UNAM, México.

Chow, Gregory (1960), “Tests of Equality between Sets of Coefficients in Two Linear Regressions", Econometrica, vol. 28, núm. 3, pp. 591-605. 
COnACyt, "Informe General del Estado de la Ciencia y la Tecnología. Informes varios de 1995 a 2014", Consejo Nacional de Ciencia y Tecnología, México.

Corona, Leonel (2004), "La tecnología, siglos xvi al xx", en Semo, Enrique (coord.), Historia económica de México, Universidad Nacional Autónoma de México/ Océano, México.

Dutrénit, Gabriela; Capdevielle, Mario; Corona Alcantar, Juan Manuel; Puchet Anyul, Martín; Santiago, Fernando y Vera-Cruz, Alexandre O. (2010), El sistema nacional de innovación mexicano: Instituciones, políticas, desempeño y desafíos, Universidad Autónoma Metropolitana-Xochimilco, México.

Freeman, Christopher (1987), Technological Policy and Economic Performance: Lessons from Japan, Pinter, London.

García, Alejandro y Heijs, Joost (2012), "La política de ciencia y tecnología en México: una síntesis de las etapas, características e instrumentos”, en Ixkic; Caballero y Guerrero (eds.), Retos globales y locales de las Ciencias Sociales, Universidad Autónoma del Estado de Morelos, Morelos, pp. 117-131.

Gauss, Susan (2011), Made in Mexico: Regions, the Nation, and the State in the Rise of Mexican Industrialism, 1920s-1940s, Penn State University Press.

Griliches, Zvi (1992), "Estadísticas de patentes como indicadores económicos: una panorámica”, EKONOMIAZ, Revista Vasca de Economía, Gobierno Vasco-Eusko Jaurlaritza-Basque Government, 23 (02), pp. 265-320.

Grossman, Gene y Helpman, Elhanan (1991), Innovation and Growth in the Global Economy, MIT Press, Cambridge.

Guzmán Chávez, Alenka; López-Herrera, Francisco y Venegas-Martínez, Francisco (2012), "Un análisis de cointegración entre patentes y crecimiento económico en México, 1980-2008”, Investigación Económica, 71-281, pp. 83-115.

Hernández Montaño, Sergio y Díaz González, Eliseo (2007), "La producción y el uso del conocimiento en México y su impacto en la innovación: análisis regional de las patentes solicitadas", Análisis Económico, vol. 22. núm. 50, pp.185-217.

IMPI (2016), “IMPI en cifras. Informes anuales, 1995-2016”, Instituto Mexicano de la Propiedad Industrial-Dirección Divisional de Promoción y Servicios de Información Tecnológica, México. (1940-1970), "Gaceta de la Propiedad Industrial. Publicaciones anuales", Instituto Mexicano de la Propiedad Industrial-Servicios de Publicación, México.

Kuntz, Sandra (2010), Historia económica general de México: de la Colonia a nuestros días, El Colegio de México-Secretaría de Economía, México.

Kuznets, Simon (1930), Secular Movements in Production and Prices. Their Nature and Their Bearing Upon Cyclical Fluctuations, Boston.

Kwiatkowski, Denis; Phillips, Peter; Schmidt, Peter y Shin, Yongcheol (1992), "Testing the Null Hypothesis of Stationarity against the Alternative of a Unit Root", Journal of Econometrics, vol. 54, núm.1-3, pp. 159-178. 
Márquez, Graciela (2014), Claves de la historia económica de México. El desempeño de largo plazo (siglos XVI-XXI), Colección Biblioteca Mexicana, Serie Historia, Fondo de Cultura Económica-Conaculta, México.

Mendoza Solís, Vandari Manuel (2014), Las patentes de invención mexicanas. Instituciones, actores y artefactos (1821-1911), tesis doctoral, El Colegio de Michoacán, México.

Mokyr, Joel (1977), "Demand versus Supply in the Industrial Revolution", Journal of Economic History, 37 (4), pp. 981-1008.

Moreno-Brid, Juan Carlos y Ros, Jaime (2009), Development and Growth in the Mexican Economy: A Historical Perspective, Oxford University Press, Oxford.

North, Douglass (2005), Understanding the Process of Economic Change, Princenton University Press.

OEMP (2006), “Clasificación internacional de patentes. Versión 2006”, página web de la OEMP: http://cip.oepm.es/descargas/20060101

OEP (2015), Reporte anual. Oficina Europea de Patentes, Alemania, página web de la OEP: http://www.epo.org/about-us/annual-reports-statistics.html

OMPI (2017), "Base de datos estadísticos de la OMPI", recuperado en febrero de 2017 en https://www.wipo.int/ipstats/es

(1983), 100 Years Protection of Industrial Property Statistics, Organización Mundial de la Propiedad Intelectual, Oficina de Publicaciones, Génova.

Ortíz-Villajos, José María (1999), Tecnología y desarrollo económico en la historia contemporánea. Estudio de las patentes registradas en España entre 1882 y 1935, OEMP, Madrid.

Pavitt, Keith (1985), "Technology Transfer among the Industrially Advanced Countries: An Overview", en Rosenberg, Nathan y Frischtadk, Claudio (eds.), International Technology Transfer: Concepts, Measures and Comparisons, New York. (1984), "Patent Statistics as Indicators of Innovative Activities: Possibilities and Problems", Scientometrics, 7 (1-2), pp. 77-99.

Quandt, Richard(1960), "Tests of the Hypothesis that a Linear Regression System Obeys Two Separate Regimes", Journal of the American Statistical Association, 55 (290), pp. 324-330.

Romer, Paul (1990), “El cambio tecnológico endógeno", El Trimestre Económico. 58 (3), 231, pp. 441-479.

Saiz, Patricio (2002), “The Spanish Patent System (1770-1907)", History of Techno$\operatorname{logy} .24, \mathrm{pp} .45-79$.

(1999), Invención, patentes e innovación en la España contemporánea, OEPM, Madrid.

Schettino, Macario (2007), Cien años de confusión. México en el siglo XX, Taurus, México.

Schmookler, Jacob (1966), Invention and Economic Growth, Cambridge. 
Shadlen, Kenneth (2012), "The Mexican Exception: Patents and Innovation Policy in a Non-Conformist and Reluctant Middle Income Country", European Journal of Development Research, vol. 24, núm. 2, pp. 300-318.

Sokoloff, Kenneth (1988), "Inventive Activity in Early Industrial America: Evidence From Patent Records, 1790-1846”, The Journal of Economic History, 48 (4), pp. 813-850.

Villavicencio, Daniel (2012), "Incentivos a la innovación en México: entre políticas y dinámicas sectoriales", en Carrillo, Jorge; Hualde, Alfredo y Villavicencio, Daniel (coords.), Dilemas de la innovación en México. Dinámicas sectoriales, territoriales e institucionales, Colegio de la Frontera Norte y Red Temática Complejidad, México, (cap. 2). 


\section{ANEXO}

Anexo 1. Datos anuales de patentes.

\begin{tabular}{|c|c|c|c|c|c|c|c|}
\hline Año & Patentes & Año & Patentes & Año & Patentes & Año & Patentes \\
\hline 1940 & 1106 & 1961 & 4477 & 1982 & 5313 & 2003 & 12197 \\
\hline 1941 & 1143 & 1962 & 4775 & 1983 & 4591 & 2004 & 13198 \\
\hline 1942 & 1180 & 1963 & 4861 & 1984 & 4459 & 2005 & 14435 \\
\hline 1943 & 4277 & 1964 & 2313 & 1985 & 3681 & 2006 & 15505 \\
\hline 1944 & 1499 & 1965 & 5966 & 1986 & 3700 & 2007 & 16599 \\
\hline 1945 & 1814 & 1966 & 6532 & 1987 & 4251 & 2008 & 16581 \\
\hline 1946 & 3022 & 1967 & 7262 & 1988 & 4400 & 2009 & 14281 \\
\hline 1947 & 2098 & 1968 & 14927 & 1989 & 4574 & 2010 & 14576 \\
\hline 1948 & 1826 & 1969 & 8227 & 1990 & 5061 & 2011 & 14055 \\
\hline 1949 & 1819 & 1970 & 8046 & 1991 & 5271 & 2012 & 15314 \\
\hline 1950 & 1876 & 1971 & 6408 & 1992 & 7695 & 2013 & 15444 \\
\hline 1951 & 2084 & 1972 & 8420 & 1993 & 8212 & 2014 & 16135 \\
\hline 1952 & 2482 & 1973 & 3853 & 1994 & 9944 & & \\
\hline 1953 & 2639 & 1974 & 2941 & 1995 & 5234 & & \\
\hline 1954 & 2803 & 1975 & 4127 & 1996 & 6754 & & \\
\hline 1955 & 2844 & 1976 & 7792 & 1997 & 3962 & & \\
\hline 1956 & 3088 & 1977 & 3454 & 1998 & 10893 & & \\
\hline 1957 & 3085 & 1978 & 4493 & 1999 & 12110 & & \\
\hline 1958 & 3411 & 1979 & 5174 & 2000 & 13061 & & \\
\hline 1959 & 3794 & 1980 & 5472 & 2001 & 13565 & & \\
\hline 1960 & 4113 & 1981 & 5997 & 2002 & 13062 & & \\
\hline
\end{tabular}

A partir de 1998 incluye las patentes registradas vía PCT. 
